\title{
Article \\ Convolution Properties of Certain Classes of Analytic Functions Defined by Jackson $q$-Derivative
}

\author{
Abdel Moneim Y. Lashin ${ }^{1,2} \mathbb{D}$, Badriah Maeed Algethami ${ }^{1, *(\mathbb{D})}$ and Abeer O. Badghaish ${ }^{1}(\mathbb{D}$ \\ 1 Department of Mathematics, Faculty of Science, King Abdulaziz University, \\ P.O. Box 80203, Jeddah 21589, Saudi Arabia; aylashin@mans.edu.eg (A.M.Y.L.); \\ abadghaish@kau.edu.sa (A.O.B.) \\ 2 Department of Mathematics, Faculty of Science, Mansoura University, Mansoura 35516, Egypt \\ * Correspondence: bmalgethami@kau.edu.sa
}

check for

updates

Citation: Lashin, A.M.Y.; Algethami, B.M.; Badghaish, A.O. Convolution Properties of Certain Classes of Analytic Functions Defined by Jackson $q$-Derivative. Mathematics 2022, 10, 105. https://doi.org/ $10.3390 /$ math10010105

Academic Editors: Valer-Daniel Breaz and Ioan-Lucian Popa

Received: 8 December 2021

Accepted: 27 December 2021

Published: 29 December 2021

Publisher's Note: MDPI stays neutral with regard to jurisdictional claims in published maps and institutional affiliations.

Copyright: (๑) 2021 by the authors Licensee MDPI, Basel, Switzerland. This article is an open access article distributed under the terms and conditions of the Creative Commons Attribution (CC BY) license (https:// creativecommons.org/licenses/by/ $4.0 /)$.

\begin{abstract}
In this paper, the Jackson $q$-derivative is used to investigate two classes of analytic functions in the open unit disc. The coefficient conditions and inclusion properties of the functions in these classes are established by convolution methods.
\end{abstract}

Keywords: analytic functions; convolution; Jackson $q$-derivative

MSC: 30C45; 30C50; 30C55; 30C80

\section{Introduction}

Let $\mathcal{B}$ be the class of functions that are analytic and of the form:

$$
f(z)=z+\sum_{k=2}^{\infty} a_{k} z^{k}, \quad(z \in \mathbb{E}:=\{z \in \mathbb{C}:|z|<1\}) .
$$

The Hadamard product (or convolution ) of two functions $f, g \in \mathcal{B}$, denoted by $f * g$, is defined by

$$
(f * g)(z)=f(z) * g(z)=z+\sum_{k=2}^{\infty} a_{k} b_{k} z^{k}
$$

where $f$ is given by (1) and $g(z)=z+\sum_{k=2}^{\infty} b_{k} z^{k}$. In 1978, Silverman et al. [1] obtained characterizations of convex, starlike and spiral-like functions in terms of convolutions. For each of these classes $\chi$, they determined a function $g$ that depends on $\chi$, such that $\frac{1}{z}(f * g) \neq 0$. both fundamental and adequate for $f$ to be in $\chi$. As a result of the work by Silverman et al. [1], many important properties of certain subclasses of analytic functions were studied by various authors (for related works, one may refer to [2-8]). For $q \in(0,1)$, the Jackson $q$-derivative of a function $f \in \mathcal{B}$ is given by $($ see $[9,10])$

$$
D_{q} f(z)= \begin{cases}\frac{f(q z)-f(z)}{(q-1) z} & (z \neq 0), \\ f^{\prime}(0) & (z=0) .\end{cases}
$$

Thus, from (2), we have

$$
D_{q} f(z)=1+\sum_{k=2}^{\infty}[k]_{q} a_{k} z^{k-1}
$$

where

$$
[n]_{q}=\frac{1-q^{n}}{1-q}
$$


and, as $q \rightarrow 1^{-},[n]_{q} \rightarrow n$. Here we used

$$
D_{q}\left(z^{n}\right)=\frac{q^{n}-1}{q-1} z^{n-1}=[n]_{q} z^{n-1}(n \in \mathbb{N}:=\{1,2,3, \ldots\}),
$$

and

$$
\lim _{q \rightarrow 1^{-}} D_{q}\left(z^{n}\right)=n z^{n-1}=\frac{d}{d z} z^{n} .
$$

Moreover, we have the following $q$-derivative rules

$$
D_{q}[f(z) g(z)]=g(z) D_{q} f(z)+f(q z) D_{q} g(z)
$$

and

$$
D_{q} \frac{f(z)}{g(z)}=\frac{g(z) D_{q} f(z)-f(z) D_{q} g(z)}{g(z) g(q z)} .
$$

We recall a known differential operator $\Omega_{q}^{n}(z)$, which was introduced by Govindaraj and Sivasubramanian (see [11]), and is also known as the Salagean $q$-differential operator, defined recursively on $n \in \mathbb{N}_{0}:=\mathbb{N} \cup\{0\}$ as follows: For $f \in \mathcal{B}$ and $q \in(0,1)$,

$$
\Omega_{q}^{0} f(z):=f(z), \Omega_{q}^{1} f(z):=z D_{q} f(z), \ldots, \Omega_{q}^{n} f(z):=z D_{q}\left(\Omega_{q}^{n-1} f(z)\right) .
$$

Then, we find that

$$
\Omega_{q}^{n} f(z)=\left(f * G_{q}^{n}\right)(z) \quad\left(n \in \mathbb{N}_{0}, f \in \mathcal{B}\right),
$$

where

$$
G_{q}^{n}(z):=z+\sum_{k=2}^{\infty}[k]_{q}^{n} z^{k}\left(n \in \mathbb{N}_{0}, z \in \mathbb{E}\right)
$$

Definition 1. A function $f \in \mathcal{B}$ is said to be in the class $\mathcal{S}_{\lambda, q}^{*}(A, B)$ if and only if

$$
(1-\lambda) \frac{f(z)}{z}+\lambda D_{q} f(z) \prec \frac{1+A z}{1+B z} \quad(z \in \mathbb{E}),
$$

where $q \in(0,1), 0 \leq \lambda \leq 1,-1 \leq B<A \leq 1, D_{q}$ is the Jackson $q$-derivative and $\prec$ denotes the usual subordination (see [12-14]).

Definition 2. A function $f \in \mathcal{B}$ is said to be in the class $\mathcal{C}_{\lambda, q}(A, B)$ if and only if

$$
D_{q} f(z)+\lambda q z D_{q}\left(D_{q} f(z)\right) \prec \frac{1+A z}{1+B z} \quad(z \in \mathbb{E}),
$$

where $q \in(0,1), 0 \leq \lambda \leq 1,-1 \leq B<A \leq 1$ and $D_{q}$ is the Jackson $q$-derivative.

With the help of the Salagean $q$-differential operator $\Omega_{q}^{n}$ given by (5), we have the following definition.

Definition 3. A function $f \in \mathcal{B}$ is said to be in the class $\mathcal{S}_{\lambda, q}^{*}(n, A, B)$ if and only if

$$
(1-\lambda) \frac{\Omega_{q}^{n} f(z)}{z}+\lambda \frac{\Omega_{q}^{n+1} f(z)}{z} \prec \frac{1+A z}{1+B z} \quad(z \in \mathbb{E}),
$$

where $q \in(0,1),-1 \leq B<A \leq 1,0 \leq \lambda \leq 1$ and $n \in N_{0}=\{0,1,2, \ldots\}$. 
In this paper, we use a technique similar to that given by Silverman et al. [1] in order to obtain certain convolution properties of the two classes $\mathcal{S}_{\lambda, q}^{*}(A, B)$ and $\mathcal{C}_{\lambda, q}(A, B)$. Furthermore, the coefficient conditions and inclusion properties of the functions in these classes are established.

\section{Convolution Conditions}

Theorem 1. Let $f(z)$ be a function of the form (1). Then $f(z) \in \mathcal{S}_{\lambda, q}^{*}(A, B)$ if and only if

$$
\frac{1}{z}\left[f(z) *\left\{(1-\lambda) \frac{z-C z^{2}}{(1-z)}+\lambda \frac{z-(q C+(1-q z) C) z^{2}}{(1-z)(1-q z)}\right\}\right] \neq 0 \quad(z \in \mathbb{E})
$$

where $C=C_{\theta}=\frac{e^{-i \theta}+A}{A-B}$, and $0 \leq \theta<2 \pi$.

Proof. Let function $f$ be in the class $\mathcal{S}_{\lambda, q}^{*}(A, B)$ if and only if

$$
(1-\lambda) \frac{f(z)}{z}+\lambda D_{q} f(z)=\frac{1+A \omega(z)}{1+B \omega(z)}
$$

which is equivalent to

$$
(1-\lambda) \frac{f(z)}{z}+\lambda D_{q} f(z) \neq \frac{1+A e^{i \theta}}{1+B e^{i \theta}}, \quad(z \in \mathbb{E}, \theta \in[0,2 \pi))
$$

which simplifies to

$$
\left[(1-\lambda) f(z)+\lambda z D_{q} f(z)\right]\left(1+B e^{i \theta}\right)-z\left(1+A e^{i \theta}\right) \neq 0 .
$$

Since

$$
f(z)=f(z) * \frac{z}{(1-z)}
$$

and

$$
D_{q} f(z)=\frac{1}{(1-z)(1-q z)} .
$$

This implies that

$$
(1-\lambda) f(z)+\lambda z D_{q} f(z)=f(z) * \frac{z-(1-\lambda) q z^{2}}{(1-z)(1-q z)} .
$$

Using (9) and (11), we get

$$
\frac{1}{z}\left[f(z) *\left\{\frac{\left(z-(1-\lambda) q z^{2}\right)\left(1+B e^{i \theta}\right)-z(1-z)(1-q z)\left(1+A e^{i \theta}\right)}{(1-z)(1-q z)}\right\}\right] \neq 0
$$

that is, that

$$
\frac{1}{z}\left[f(z) * \frac{\left.-\lambda\left\{(A-B) z e^{i \theta}-\left(q\left(1+A e^{i \theta}\right)+(1-q z)\left(1+A e^{i \theta}\right)\right) z^{2}\right\}\right]}{(1-z)(1-q z)}\right] \neq 0
$$

or, equivalently,

$$
\frac{1}{z}\left[f(z) *-(1-\lambda)\left\{\frac{z-\frac{\left(e^{-i \theta}+A\right)}{(A-B)} z^{2}}{(1-z)}\right\}-\lambda\left\{\frac{z-\left(\frac{q\left(e^{-i \theta}+A\right)}{(A-B)}+\frac{(1-q z)\left(e^{-i \theta}+A\right)}{(A-B)}\right) z^{2}}{(1-z)(1-q z)}\right\}\right] \neq 0 .
$$


Then, (12) can be rewritten as the following

$$
\frac{1}{z}\left[f(z) *\left\{(1-\lambda) \frac{z-C z^{2}}{(1-z)}+\lambda \frac{z-(q C+(1-q z) C) z^{2}}{(1-z)(1-q z)}\right\}\right] \neq 0(z \in \mathbb{E}) .
$$

Hence, the first part of Theorem 1 was proven.

Conversely, since assumption (8) holds for $C=0$, it follows that

$$
(1-\lambda) f(z)+\lambda z D_{q} f(z) \neq 0 \text { for all } z \in \mathbb{E},
$$

hence the function $\varphi(z)=(1-\lambda) f(z)+\lambda z D_{q} f(z)$ is analytic in $\mathbb{E}$ (i.e., it is regular in $z_{0}=0$, with $\varphi(0)=1$ ).

We obtain from the first part that

$$
(1-\lambda) \frac{f(z)}{z}+\lambda D_{q} f(z) \neq \frac{1+A e^{i \theta}}{1+B e^{i \theta}},(z \in \mathbb{E}, 0 \leq \theta<2 \pi) .
$$

If we denote

$$
\Psi(z)=\frac{1+A z}{1+B z},(z \in \mathbb{E}),
$$

relation (14) shows that $\varphi(\mathbb{E}) \cap \Psi(\partial \mathbb{E})=\phi$. Thus, the simply connected domain $\varphi(\mathbb{E})$ is included in a connected component of $\mathbb{C} \backslash \Psi(\partial \mathbb{E})$. From here, using the fact that $\varphi(0)=$ $\Psi(0)$ together with the univalent of the function $\Psi$, it follows that $\varphi(z) \prec \Psi(z)$; that is $f(z) \in \mathcal{S}_{\lambda, q}^{*}(A, B)$. Thus, the second part of Theorem 1 was proven.

Taking $\lambda=1$ in Theorem 1, we get the following corollary.

Corollary 1. Let the function $f$ be of the form (1). Then

$$
D_{q} f(z) \prec \frac{1+A z}{1+B z} \quad(0<q<1,-1 \leq B<A \leq 1),
$$

if and only if

$$
\frac{1}{z}\left[f(z) * \frac{z-(q C+(1-q z) C) z^{2}}{(1-z)(1-q z)}\right] \neq 0(z \in \mathbb{E})
$$

where $C=C_{\theta}=\frac{e^{-i \theta}+A}{A-B}$, and $0 \leq \theta<2 \pi$.

Letting $q \rightarrow 1^{-}$in Theorem 1 , we acquire the following corollary

Corollary 2. Let the function $f$ be of the form (1). Then,

$$
(1-\lambda) \frac{f(z)}{z}+\lambda f^{\prime}(z) \prec \frac{1+A z}{1+B z} \quad(-1 \leq B<A \leq 1),
$$

if and only if

$$
\frac{1}{z}\left[f(z) *\left((1-\lambda) \frac{z-C z^{2}}{(1-z)}+\lambda \frac{\left.z-(C+(1-z) C) z^{2}\right)}{(1-z)^{2}}\right)\right] \neq 0(z \in \mathbb{E})
$$

where $C=C_{\theta}=\frac{e^{-i \theta}+A}{A-B}$ and $0 \leq \theta<2 \pi$.

Theorem 2. A function $f(z)$ of the form (1) is in the class $\mathcal{S}_{\lambda, q}^{*}(n, A, B)$ if and only if

$$
1-\sum_{k=2}^{\infty}[k]_{q}^{n}\left(\frac{e^{-i \theta}+B}{A-B}\right)\left[(1-\lambda)+\lambda[k]_{q}\right] a_{k} z^{k-1} \neq 0
$$


for all $0 \leq \theta<2 \pi$ and $z \in \mathbb{E}$.

Proof. Note that

$$
f \in \mathcal{S}_{\lambda, q}^{*}(n, A, B) \Leftrightarrow \Omega_{q}^{n} f(z) \in \mathcal{S}_{\lambda, q}^{*}(A, B) .
$$

From Theorem 1 , we have $f \in \mathcal{S}_{\lambda, q}^{*}(n, A, B)$ if and only if

$$
\frac{1}{z}\left[\Omega_{q}^{n} f(z) *\left\{(1-\lambda) \frac{z-C z^{2}}{(1-z)}+\lambda \frac{z-(q C+(1-q z) C) z^{2}}{(1-z)(1-q z)}\right\}\right] \neq 0(z \in \mathbb{E})
$$

where $C=C_{\theta}=\frac{e^{-i \theta}+A}{A-B}$, and $0 \leq \theta<2 \pi$. Now we can easily deduce that

$$
\begin{aligned}
& \frac{1}{z}\left[\Omega_{q}^{n} f(z) *\left\{(1-\lambda) \frac{z-C z^{2}}{(1-z)}+\lambda \frac{z-(q C+(1-q z) C)) z^{2}}{(1-z)(1-q z)}\right\}\right] \\
= & \frac{1}{z}\left[\Omega_{q}^{n} f(z) *(1-\lambda)\left\{C z+\frac{z(1-C)}{(1-z)}\right\}+\lambda\left\{C z+\frac{z(1-C)}{(1-z)(1-q z)}\right\}\right] .
\end{aligned}
$$

Using

$$
\frac{z}{1-z}=z+\sum_{k=2}^{\infty} z^{k}, \frac{z}{(1-z)(1-q z)}=z+\sum_{k=2}^{\infty}[k]_{q} z^{k}
$$

and

$$
\Omega_{q}^{n} f(z)=z+\sum_{k=2}^{\infty}[k]_{q}^{n} a_{k} z^{k}
$$

(17) may be written as

$$
1+\sum_{k=2}^{\infty}[k]_{q}^{n}(1-C)\left[(1-\lambda)+\lambda[k]_{q}\right]_{k} z^{k-1}
$$

since $C=\frac{e^{-i \theta}+A}{A-B}$, then (18) gives

$$
1-\sum_{k=2}^{\infty}[k]_{q}^{n}\left(\frac{e^{-i \theta}+B}{A-B}\right)\left[(1-\lambda)+\lambda[k]_{q}\right]_{k} a^{k-1}
$$

therefore, (16) becomes

$$
1-\sum_{k=2}^{\infty}[k]_{q}^{n}\left(\frac{e^{-i \theta}+B}{A-B}\right)\left[(1-\lambda)+\lambda[k]_{q}\right]_{k} z^{k-1} \neq 0 .
$$

This finishes the proof of Theorem 2.

Theorem 3. If $f(z) \in \mathcal{B}$ satisfies the inequality

$$
\sum_{k=2}^{\infty}(1+|B|)\left[(1-\lambda)+\lambda[k]_{q}\right][k]_{q}^{n}\left|a_{k}\right| \leq(A-B)
$$

then $f(z) \in \mathcal{S}_{\lambda, q}^{*}(n, A, B)$.

Proof. Since

$$
\left|\frac{e^{-i \theta}+B}{A-B}\right| \leq \frac{\left|e^{-i \theta}\right|+|B|}{|A-B|}=\frac{1+|B|}{A-B}
$$


then,

$$
\begin{aligned}
& \left|1-\sum_{k=2}^{\infty}[k]_{q}^{n}\left(\frac{e^{-i \theta}+B}{A-B}\right)\left[(1-\lambda)+\lambda[k]_{q}\right] a_{k} z^{k-1}\right| \\
\geq & 1-\sum_{k=2}^{\infty}[k]_{q}^{n}\left|\frac{e^{-i \theta}+B}{A-B}\right|\left[(1-\lambda)+\lambda[k]_{q}\right]\left|a_{k}\right||z|^{k-1} \\
\geq & 1-\sum_{k=2}^{\infty}[k]_{q}^{n}\left(\frac{1+|B|}{A-B}\right)\left[(1-\lambda)+\lambda[k]_{q}\right]\left|a_{k}\right|>0 . \quad(z \in \mathbb{E})
\end{aligned}
$$

Thus, from Theorem 2, we have $f(z) \in \mathcal{S}_{\lambda, q}^{*}(n, A, B)$, which ends the proof.

Theorem 4. $\mathcal{S}_{\lambda, q}^{*}(n+1, A, B) \subset \mathcal{S}_{\lambda, q}^{*}(n, A, B)$.

Proof. Since $f(z) \in \mathcal{S}_{\lambda, q}^{*}(n+1, A, B)$, we see from Theorem 2 that

$$
1-\sum_{k=2}^{\infty}[k]_{q}^{n+1}\left(\frac{e^{-i \theta}+B}{A-B}\right)\left[(1-\lambda)+\lambda[k]_{q}\right] a_{k} z^{k-1} \neq 0
$$

we can write (20) as

$$
\left[1+\sum_{k=2}^{\infty}[k]_{q} z^{k-1}\right] *\left[1-\sum_{k=2}^{\infty}[k]_{q}^{n}\left(\frac{e^{-i \theta}+B}{A-B}\right)\left[(1-\lambda)+\lambda[k]_{q}\right] a_{k} z^{k-1}\right] \neq 0 .
$$

Since,

$$
\begin{aligned}
& {\left[1+\sum_{k=2}^{\infty}[k]_{q} z^{k-1}\right] *\left[1+\sum_{k=2}^{\infty} \frac{1}{[k]_{q}} z^{k-1}\right] } \\
= & 1+\sum_{k=2}^{\infty} z^{k-1}=\frac{1}{1-z} .
\end{aligned}
$$

By utilizing the property, if $f \neq 0$ and $g * h \neq 0$, then $f *(g * h) \neq 0,(21)$ can be written as

$$
1-\sum_{k=2}^{\infty}[k]_{q}^{n}\left(\frac{e^{-i \theta}+B}{A-B}\right)\left[(1-\lambda)+\lambda[k]_{q}\right] a_{k} z^{k-1} \neq 0
$$

which means that $f(z) \in \mathcal{S}_{\lambda, q}^{*}(n, A, B)$. This finishes the proof of Theorem 4 .

Theorem 5. Let the function $f$ be of the form (1). Then $f \in \mathcal{C}_{\lambda, q}(A, B)$ if and only if

$$
\frac{1}{z}\left[f(z) *\left\{C z+(1-\lambda) \frac{z(1-C)}{(1-z)(1-q z)}+\lambda \frac{z(1-C)(1+q z)}{(1-z)(1-q z)\left(1-q^{2} z\right)}\right\}\right] \neq 0
$$

where $z \in \mathbb{E}, C=C_{\theta}=\frac{e^{-i \theta}+A}{A-B}$ and $0 \leq \theta<2 \pi$.

Proof. Note that

$$
f \in \mathcal{C}_{\lambda, q}(A, B) \Leftrightarrow z D_{q} f(z) \in \mathcal{S}_{\lambda, q}^{*}(A, B) .
$$

Theorem 1 gives

$$
\frac{1}{z}\left[z D_{q} f(z) *\left\{(1-\lambda)\left\{C z+\frac{z(1-C)}{(1-z)}\right\}+\lambda\left\{C z+\frac{z(1-C)}{(1-z)(1-q z)}\right\}\right\}\right] \neq 0
$$

where $z \in \mathbb{E}, C=C_{\theta}=\frac{e^{-i \theta}+A}{A-B}$ and $0 \leq \theta<2 \pi$. Since 


$$
z D_{q}(f * g)=f(z) * z D_{q} g(z)
$$

then, (25) can be written as

$$
\frac{1}{z}\left[f(z) *\left\{(1-\lambda)\left(\frac{z(1-C)}{(1-z)(1-q z)}+C z\right)+\lambda\left(\frac{z(1-C)(1+q z)}{(1-z)(1-q z)\left(1-q^{2} z\right)}+C z\right)\right\}\right] \neq 0 .
$$

This finishes the proof of Theorem 5 .

Taking $\lambda=1$ in Theorem 5, we get the following corollary.

Corollary 3. Let the function $f$ be of the form (1). Then

$$
D_{q} f(z)+q z D_{q}\left(D_{q} f(z)\right) \prec \frac{1+A z}{1+B z} \quad(0<q<1,-1 \leq B<A \leq 1),
$$

if and only if

$$
\frac{1}{z}\left[f(z) *\left(\frac{z(1-C)(1+q z)}{(1-z)(1-q z)\left(1-q^{2} z\right)}+C z\right)\right] \neq 0(z \in \mathbb{E})
$$

where $C=C_{\theta}=\frac{e^{-i \theta}+A}{A-B}$ and $0 \leq \theta<2 \pi$.

Letting $q \rightarrow 1^{-}$in Corollary( 3 ), we acquire the following corollary.

Corollary 4. Let the function $f$ be of the form in (1). Then

$$
f^{\prime}(z)+z f^{\prime \prime}(z) \prec \frac{1+A z}{1+B z} \quad(-1 \leq B<A \leq 1),
$$

if and only if

$$
\frac{1}{z}\left[f(z) *\left(\frac{z(1-C)(1+z)}{(1-z)^{3}}+C z\right)\right] \neq 0(z \in \mathbb{E})
$$

where $C=C_{\theta}=\frac{e^{-i \theta}+A}{A-B}$ and $0 \leq \theta<2 \pi$.

\section{Conclusions}

In this paper, we introduced and studied two new subclasses of analytic functions in the open unit disc using the Jackson q-derivative. A similar technique to that given by Silverman et al. [1] was used to obtain certain convolution properties for these two classes. In addition, the coefficient conditions and inclusion properties of these classes are established. Several special cases have been examined as applications of our main results.

Author Contributions: Conceptualization, A.M.Y.L.; Funding acquisition, B.M.A.; Investigation, B.M.A.; Project administration, A.O.B.; Supervision, A.M.Y.L. and A.O.B.; Writing (original draft), B.M.A.; Writing ( review and editing), A.M.Y.L.and A.O.B. All authors have read and agreed to the published version of the manuscript.

Funding: This research was funded by the Deanship of Scientific Research (DSR) at King Abdulaziz University, Jeddah, Saudi Arabia(FP-212-43).

Institutional Review Board Statement: Not applicable.

Informed Consent Statement: Not applicable.

Acknowledgments: The authors would like to express their thanks to the referees for their helpful comments and suggestions that improved the presentation of the paper.

Conflicts of Interest: The authors declare no conflict of interest. 


\section{References}

1. Silverman, H.; Silvia, E.M.; Telage, D. Convolution conditions for convexity, starlikeness and spiral-likeness. Math. Zeitschr. 1978, 162, 125-130. [CrossRef]

2. Seoudy, T.M.; Aouf, M.K. Convolution properties for certain classes of analytic functions defined by q-derivative operator. Abstr. Appl. Anal. 2014, 2014, 846719. [CrossRef]

3. Aouf, M.K.; Seoudy, T.M. Convolution properties for classes of bounded analytic functions with complex order defined by q-derivative operator. Rev. Real Acad. Cienc. Exactas Ser. A. Mat. 2019, 113, 1279-1288. [CrossRef]

4. Lashin, A.Y. Convolution conditions for bounded $\alpha$-starlike functions of complex order. Ann. Univ. Marie Curie-Sklodowska Sect. A 2017, 71, 65-72. [CrossRef]

5. Khan, M.G.; Ahmad, B.; Khan, R.; Zubair, M.; Salleh, Z. On q-analogue of Janowski-type starlike functions with respect to symmetric points. Demonstr. Math. 2021, 54, 37-46. [CrossRef]

6. Piejko, K.; Sokół, J. On convolution and q-calculus. Bol. Soc. Mat. Mex. 2020, 26, 349-359. [CrossRef]

7. Piejko, K.; Sokól, J.; Trabka-Wieclaw, K. On q-Calculus and Starlike Functions. Iran J. Sci. Technol. Trans. Sci. 2019, 43, 2879-2883. [CrossRef]

8. Srivastava, H.M.; Arif, M.; Raza, M. Convolution properties of meromorphically harmonic functions defined by a generalized convolution q-derivative operator. AIMS Math. 2021, 6, 5869-5885. [CrossRef]

9. Jackson, F.H. On $q$-functions and a certain difference operator. Trans. R. Soc. Edinb. 1908, 46, 253-281. [CrossRef]

10. Jackson, F.H. On q-definite integrals. Q. J. Pure Appl. Math. 1910, 41, 193-203.

11. Govindaraj, M.; Sivasubramanian, S. On a class of analytic functions related to conic domains involving $q$-calculus. Anal. Math. 2017, 43, 475-487. [CrossRef]

12. Miller, S.S.; Mocanu, P.T. Differential Subordination: Theory and Applications; Series on Monographs and Textbooks in Pure and Applied Mathematics; Marcel Dekker Inc.: New York, NY, USA; Basel, Switzerland, 2000; Volume 255.

13. Aouf, M.K.; Mostafa, A.O.; Seoudy, T.M. Subordination and Superordination Results for Analytic Functions Involving Certain Operators; Lambert Academic Publishing: Chisinau, Republic of Moldova, 2014.

14. Bulboacã, T. Differential Suborinations and Superordinations, Recent Results; House of Scientific Book Publishing: Cluj-Napoca, Romania, 2005. 